\title{
L'AUTOREPRÉSENTATION EN BANDE DESSINÉE, AUTOPORTRAIT OU AUTOBIOGRAPHIE
}

\author{
Sylvain RHEAULT, Université de Regina
}

Quand on pense aux genres populaires en bande dessinée, l'aventure à la Tintin et l'humour comme celui d'Astérix viennent en tête de liste. Outre ces genres qui ont fait la fortune des grandes maisons d'édition, d'autres séries populaires offrent des enquêtes policières, comme Inspecteur Canardo, de la science fiction, comme Valérian, de l'horreur, comme Adèle Blanc-sec que Luc Besson a porté à l'écran en 2010, ou de l'érotisme, dont Barbarella est sans doute l'exemple le plus connu. Cependant, depuis un certain temps déjà, on trouve dans le catalogue des petites maisons d'édition indépendantes des bandes dessinées qui offrent des genres encore peu explorés, dont l'autobiographie. En effet, pour le ou la bédéiste qui doit composer à la fois avec du texte et du dessin, le genre autobiographique en bande dessinée offre certainement des défis curieux et fascinants. Le défi en est aussi un de taille pour le critique. En effet, l'autoreprésentation en bande dessinée relève-t-elle de l'autoportrait ou de l'autobiographie ? C'est la question à laquelle nous allons tenter de répondre dans le présent article. Nous nous servirons du Journal (1) de Fabrice Neaud pour contraster les théories de l'autobiographie avec celles de l'autoportrait. Nous analyserons les caractéristiques les plus remarquables de l'œuvre pour voir auquel des deux genres s'apparente le plus l'autoreprésentation en BD.

\section{Un « journal »}

Journal (1) raconte environ 18 mois de la vie de Fabrice Neaud, un jeune artiste homosexuel au chômage. Ce dernier présente ses amis et ses amants potentiels, décrit ses activités quotidiennes et ses émotions. Journal (1) est le premier d'une série de quatre albums. Celui-ci fait 112 pages alors que les suivants font respectivement 71, 431 et 224 pages. Il s'agit donc d'un ensemble plutôt substantiel. Journal (1) a pour sous-titre «février 1992 - septembre 1993 ». Ces terminus post quem et ante quem encadrent l'histoire des amours malheureuses de l'auteur pour Stéphane. Mais revenons au titre. Les 
premières autobiographies de la civilisation chrétienne portaient le nom de « confessions », titre qu'on trouve apposé entre autres sur des textes de St-Augustin et de Rousseau. Ces projets littéraires apparaissaient alors comme des occasions d'avouer ses fautes et de réfléchir à la faiblesse humaine. Dans le cas de Neaud, l'œuvre a pour titre «journal », ce qui suggère plutôt l'écriture du quotidien. Neaud développe longuement les angoisses que lui cause sa solitude ainsi que ses tentatives infructueuses de trouver un compagnon stable. C'est que les hommes qui plaisent à Neaud, ont pour défaut d'être volages, en particulier Stéphane. D’autre part, Neaud se remémore des événements clés de son enfance, dont certains lui sont douloureux et lui font faire des cauchemars. Philippe Lejeune, dans Le Pacte autobiographique (1996, 14), définit l'autobiographie comme « un récit rétrospectif en prose qu'une personne réelle fait de sa propre existence, lorsqu'elle met l'accent sur sa vie individuelle, en particulier sur l'histoire de sa personnalité ». Cette définition, citée des milliers de fois, semble convenir parfaitement au sujet de notre étude et rapproche l'autoreprésentation en bande dessinée de l'autobiographie. On peut même trouver dans Journal (1) un moignon de pacte autobiographique lorsqu'un ami de Neaud lui conseille, pour la bande dessinée qu'il est en train de réaliser: « ... pas de censure, parle de tout, ne déguise rien » (29). Le journal, pour celui qui le tient, est justement le lieu d'un dévoilement sans restriction, puisque les sentiments fraîchement vécus n'ont pas encore été filtrés, et que l'effet des gaffes sentimentales reste encore vif. Neaud n'hésite pas à se représenter en train de se masturber (23). Ce n'est pas la première fois qu'on a ce genre de scène en bande dessinée. Mais la visée ici n'est pas de provoquer le scandale à tout prix. Il faut plutôt voir dans ce geste exhibitionniste la résolution indéfectible de l'artiste à tout raconter sur lui, fussent les détails scabreux. Ce genre d'aveu contribue à renforcer le pacte autobiographique établi au départ avec le lecteur.

\section{L'espace de l'homosexualité}

Les artistes peintres qui se sont adonnés à l'autoportrait ont établi au fil des siècles de nombreuses conventions picturales. Étant peintre, c'est comme tel que l'artiste se représente et l'atelier est ainsi devenu l'un des cadres favoris pour l'autoportrait. On peut penser à l'Atelier du peintre (1855) de Gustave Courbet, l'un des exemples les plus 
célèbres. Ce qui étonne, dans cette œuvre, c'est combien peu de place occupe l'artiste dans l'ensemble de la gigantesque toile. Courbet semble vouloir définir son identité non pas tant en brossant dans les détails ses propres traits physiques mais plutôt en mettant en scène le lieu, les objets et les personnes qui composent la mosaïque de sa vie d'artiste. «[Courbet] personnifie la vision du peintre, l'atelier encombré symbolisant le monde qui l'entoure » (Koortbojian 51). S'il fallait rapprocher le travail de Neaud de l'autoportrait, c'est avec les lieux qu'il faudrait commencer. Neaud illustre sa chambre, bien sûr, l'endroit où il vit, où il travaille, où il a ses cauchemars, et aussi où il reçoit à l'occasion ses amants. La chambre symbolise l'espace intérieur où se construit l'identité mais où règne aussi une solitude obsédante. C'est pourquoi Neaud passe beaucoup de temps à tenter d'en sortir et fréquente ce qu'on pourrait désigner comme les lieux de l'homosexualité. Avec le personnage narrateur, on assiste aux rencontres furtives du jardin public, la nuit (18). Les toilettes publiques, les buissons, les voitures garées créent un univers de désirs et de secrets où il faut attendre des heures et des heures, plusieurs jours de suite, dans l'espoir de dénicher l'âme sœur. Mais on sent de pair avec l'espoir une peur poignante et on apprendra que Neaud s'est fait agresser dans le parc (81). Les longues heures de drague contribuent sans doute à créer un effet de réalisme mais aussi rendent encore plus précieuse la rencontre avec Stéphane. Et puis il y a ces bars réservés aux homosexuels où Neaud passe de longues soirées, avec leur faune particulière (41) que Neaud catégorise avec soin. C'est qu'il n'y trouve pour ainsi dire jamais de compagnons correspondant spécifiquement à ses fantasmes personnels, soit des hommes musclés aux attributs corporels hyper-masculinisés. Stéphane, évidemment, tout au long de l'album, constitue l'unique exception à ces attentes irréalistes. Pour en revenir à l'espace, la bande dessinée, on le constate, permet de multiplier les explorations de lieux, ce que ne peut même tenter la peinture. Au moyen d'une succession de «tableaux », Neaud brosse un panorama de son espace extérieur, c'est-à-dire le lieu de rencontre de l'autre.

\section{La représentation des corps}

Il serait difficile pour un/une bédéiste de se représenter avec autant de détails que le fait l'artiste sur une toile. En effet, comme le médium de la bande dessinée impose de 
redessiner son personnage d'une case à l'autre, il faut en arriver à un compromis acceptable entre la représentation et l'économique du trait. L'artiste de bande dessinée doit donc composer avec une contrainte qualitative. Voilà qui nous éloigne de l'autoportrait. Cependant, il y a l'utilisation des poses. Les toutes premières pages du Journal (1) montrent Neaud enfant en train d'être tabassé et déculotté par des garçons au centre d'une clairière. La dernière case du malheureux épisode d'agression montre l'enfant gisant en position fœtale, traumatisé par la violence dont il vient d'être victime. Il s'agit là d'une position récurrente dans l'album, entre autres aux pages 13, 19, 87, 102 et 104. La page 87, en particulier, montre Neaud sur toute la planche en position fotale inversée. Il vient de subir un autre cuisant revers amoureux. Michael Koorbojian (1992, $45)$ et Laura Cumming $(2009,100)$ expliquent comment les poses dans les portraits et les autoportraits symbolisent des idées, comme la fidélité, ou réfèrent subtilement à d'autres œuvres. Ainsi, au-delà de la simple représentation, l'autoportrait, du moins dans ses expressions classiques, s'applique aussi à créer des effets de référence. La pose symbolique n'est pas un phénomène nouveau en bande dessinée. Goscinny et Uderzo, dans la série Astérix, en usent et abusent pour obtenir des effets comiques. Il suffit de penser aux diverses poses qu'adopte l'esclave musclé dans les premières pages de l'album Les Lauriers de César. Cependant Neaud innove, du moins aux yeux du lecteur hétérosexuel que nous sommes, en inscrivant les poses corporelles dans un registre homosexuel. Le plus frappant est sans doute le désir du toucher tendre, qu'il s'agisse de toucher l'autre ou de se faire toucher par lui. Avec le regard dont nous parlerons plus loin, le toucher apparaît comme l'une des sensations les plus fréquemment sollicitées. Le désir du toucher tendre prend la forme visuelle d'une main masculine tendue vers l'autre (57), main inspirée de celle de Dieu créant Adam (57), qu'on peut voir dans la fameuse scène de la genèse de la chapelle Sixtine peinte par Michelangelo. Le choix n'est pas gratuit, Michelangelo étant connu pour sa passion du corps masculin, que d'aucuns qualifient d'homoérotique. Cette main tendue vers l'autre réapparaîtra lorsque le désir surgira à nouveau $(61,78,79)$ pour se rétracter lorsque s'esquive l'objet du désir. Notons que la main tendue parvient à toucher « directement » le cœur de Neaud (61), mêlant de manière étonnante deux métaphores visuelles. Malgré son fantasme pour des corps hypermasculins, c'est avant tout une relation intime que recherche Neaud. Notons enfin que le 
désir du toucher tendre se voit exacerbé par les tabous sociaux. Il n'est permis aux homosexuels de se toucher tendrement qu'en certains lieux, comme la chambre et les bars homosexuels. Au jardin public, c'est possible, mais cela reste une entreprise risquée, comme l'a appris Neaud à ses dépens. Partout ailleurs, il vaut mieux respecter l'interdit social. L'analyse nous a fait parcourir beaucoup de chemins parallèles et il faut se recentrer. Nous conclurons ce paragraphe en rappelant que l'utilisation de symboles chez Neaud semble emprunter beaucoup aux techniques classiques de l'autoportrait.

\section{Le regard}

Un autre aspect remarquable de l'œuvre de Neaud, c'est la mise en scène de son propre regard, en particulier ce regard que porte le personnage narrateur sur les hommes qu'il convoite. Des parties du corps de ces hommes sont mises en gros plan dans une case, comme un cou mal rasé (95), ce qui leur confère une connotation érotique. Neaud tire d'ailleurs plusieurs portraits d'hommes, en photo comme en peinture, dont celui de Stéphane. Pour Neaud, porter son regard sur l'autre, c'est l'inviter à la rencontre. Neaud s'approprie l'image de celui qui accepte de lui parler et considère dorénavant cette image comme faisant partie de son identité propre. Il vaut sans doute la peine de rapporter qu'une certaine controverse entoure la publication des albums de la série Journal : Neaud les aurait publiés sans demander la permission à certaines des personnes qui y figurent d'utiliser leur image (Paquin 4-5). Il y aurait même eu des menaces de poursuite. Cela prouve-t-il que l'autobiographe est prêt à assumer toutes les conséquences de son pacte autobiographique ? Ou peut-être que l'autoportraitiste ne peut-il achever la réalisation de son identité que dans le reflet qu'il trouve chez les autres ? Mais revenons-en au regard. Il y a ces remarquables planches montrant onze vues de dos de Patrick (108-109) où soit le cadrage, soit l'ombre, soit la qualité du trait varie subtilement avec chacune des répliques que s'échangent le narrateur et l'objet de son désir. Cela a pour effet de représenter visuellement les coups émotionnels ressentis par le narrateur. À deux moments, sous l'intensité des paroles de Stéphane, les traits deviennent embrouillés, comme si des larmes montaient aux yeux du narrateur. Il est remarquable que Neaud, dans ces cases, occupe la position du public, comme si nous regardions Stéphane avec lui. Lorsque Neaud demande à Stéphane de le regarder (77), c'est plutôt avec les yeux du lecteur que 
ceux de Stéphane établissent un contact. Neaud fait ainsi du lecteur son complice malgré lui. Cependant, le regard change lorsqu'il est braqué sur le personnage de Neaud. Le narrateur perçoit son corps de manière négative et semble se complaire à mettre en évidence la maigreur de son anatomie (87). Le corps maigrelet du personnage de Neaud se voit d'autant plus déprécié qu'il est mis en contraste avec ceux de ses fantasmes : des corps d'hommes musclés, comme ceux de Bruce Willis (49) et de Mel Gibson ou Ed Harris (23). Notons que Patrick apparaît comme un homme au corps bien charpenté (40), conforme à l'idéal fantasmatique de l'artiste. L'autoportrait, encore une fois, semble le plus proche parent de l'autoreprésentation.

\section{Le style}

L'autoreprésentation, en bande dessinée, ne consiste pas, pour l'artiste, à se dessiner de la manière la plus réaliste possible, loin de là. D'ailleurs, Chris Ware, un auteur américain, a publié certaines de ses œuvres sans même y apposer sa signature, ou bien en minimisant cette dernière. Ware affirme dans une interview retranscrite dans Chris Ware : La bande dessinée réinventée que son style suffit à le faire reconnaître. «Le style, c'est l'homme » disait déjà Buffon en 1753, et, en effet, ne reconnaît-on pas tout de suite les pastels de Chardin ou les traits nerveux de Van Gogh ? En bande dessinée, le grand public n'a-t-il pas assimilé la ligne claire d'Hergé dans les albums de Tintin ou le trait rond d'Uderzo dans un dessin d'Astérix ? Pour en revenir aux représentations que Neaud fait de lui-même, lorsqu'on les compare à des photos, on constate que les traits sont peu conformes au modèle réel. Tout comme pour l'autobiographie, en fait, l'autoreprésentation trouve d'abord son expression dans le caractère unique du style de Neaud, fait de traits nerveux et de hachurés. La griffe personnelle de l'artiste se révèle ainsi la plus fiable des méthodes d'autoreprésentation.

\section{En guise de conclusion}

Après ce survol des caractéristiques du Journal (1) de Neaud, peut-on maintenant élaborer une réponse au problème posé initialement, à savoir si l'autoreprésentation en bande dessinée relève davantage de l'autoportrait ou de l'autobiographie ? Un peu des deux, serait-on tenté de proposer comme arrangement à l'amiable. Pourtant, malgré 
quelques points de ressemblance, il faut avouer que ni les théories de l'autoportrait, ni celle de l'autobiographie ne donnent entièrement satisfaction. Il faudrait plutôt, comme le propose Thierry Groensteen, élaborer pour la bande dessinée une méthode critique propre. Il faudrait, pour aborder l'autoreprésentation, disposer de théories capables d'intégrer la narration et la représentation. Les approches multidisciplinaires comme celle que nous venons de tenter, ne sont pas inutiles. Elles permettent de cerner les limites des médias. Nul doute que les recherches à venir dans le domaine de l'autoreprésentation seront fructueuses et pourront apporter un éclairage nouveau autant sur l'autobiographie que sur l'autoportrait.

\section{Ouvrages cités}

Beaty, Bart. UNpopular Culture. Toronto : U of Toronto P, 2007.

Cumming, Laura. A Face to the World. London : HarperPress, 2009.

Groensteen, Thierry. Système de la bande dessinée. Paris : PUF, 1999.

Koortbojian, Michael. Autoportraits. Paris: Réunion des musées Nationaux, 1992.

Lejeune, Philippe. Le Pacte autobiographique (1975). Paris : Seuil, 1996.

---. Signes de vie. Paris : Seuil, 2005.

Neaud, Fabrice. Journal (1) février 1992 - septembre 1993 (1996). Angoulème : Ego comme X, 2003.

McCloud, Scott. Reinventing Comics. New York : Harper Collins, 2000.

Paquin, Éric. « Autobiographie dessinée ». Spirale 197 (2004) : 4-5.

Peeters, Benoît. Samson, Jacques. Chris Ware : La bande dessinée réinventée. Belgique : Impressions nouvelles, 2009.

Ware, Chris. Jimmy Corrigan. Seattle : Fantagraphic, 2000. 